\title{
A NEW APPROACH TO THE MICROSTRIP LINES WITH FINITE STRIP THICKNESS AND CONDUCTIVITY
}

\author{
Jeng-Yi Ke and Chun Hsiung Chen \\ Department of Electrical Engineering, \\ National Taiwan University, \\ Taipei, Taiwan 10617, ROC
}

\begin{abstract}
A new approach is proposed for an analysis of microstrip line whose signal strip has finite thickness and conductivity. By this approach, the CPU time in computation may be much reduced. In this work, the phase constant $\beta$ and attenuation constant $\alpha$ of a microstrip line with finite strip thickness and finite conductivity are discussed, together with the current distributions along the signal strip.
\end{abstract}

\section{Introduction}

Previous analysis of microwave planar waveguiding structures is usually conducted under the assumptions of infinitely thin strip and infinite conductivity. In MMIC, however, the problem of finite thickness and finite conductivity must be taken into consideration, because the strip thickness may be comparable to the strip width.

The characteristics of microstrip lines with finite strip thickness and conductivity were discussed by the techniques such as the perturbation approach [1] and the full-wave techniques of mode matching [2] and method of lines [3]. However, the perturbation approach is not suitable for MMIC in which the skin depth and the strip thickness are of the same order, and the above-mentioned fullwave techniques are time consuming. Therefore, an easier and faster method should be needed to meet the development of MMIC.

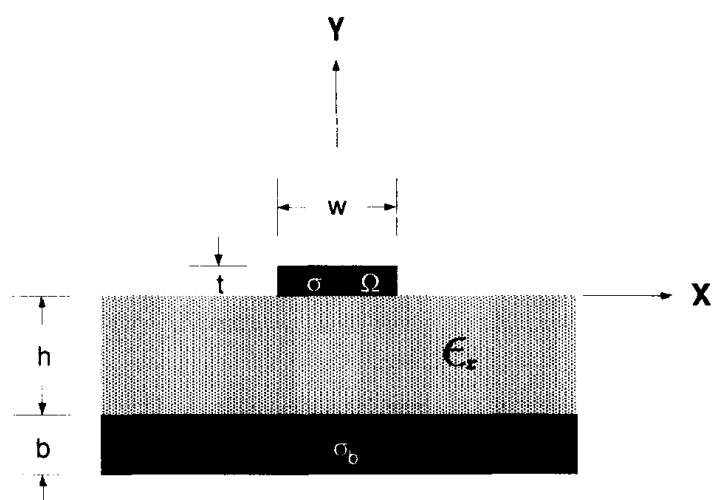

Figure 1: Cross section of microstrip line with layer structure.

In this study, a new method is proposed to deal with the microstrip line with layer structure in which the thickness and conductivity of signal strip are finite. Here, the spectral domain approach (SDA) is modified to adapt the case with two-dimensional current distributions. By this new method, the integration along one direction is analytically integrated. Hence it is as easy as the conventional SDA in which only one-dimensional integration has to be performed to get the matrix equation for the propagation constant.

\section{Formulation}

The cross section of the microstrip line with layer structure is shown in Fig. 1. The conductivity of the signal strip is $\sigma$ and the thickness is $t$. The relation between the electric field $\mathbf{E}(\mathbf{r})$ and 
the current density $\mathbf{J}(\mathbf{r})$ within the strip conductor $\Omega$ is

$$
\mathbf{E}(\mathbf{r})=\int_{\Omega} \overline{\overline{\mathbf{G}}}\left(\mathbf{r}-\mathbf{r}^{\prime}\right) \bullet \mathbf{J}\left(\mathbf{r}^{\prime}\right) \mathbf{d} \mathbf{r}^{\prime}=\frac{\mathbf{1}}{\sigma} \mathbf{J}(\mathbf{r})
$$

By weighting both sides of (1) by an arbitrary function $\mathbf{w}(\mathbf{r})$ and then integrated, one may get an integral equation involving $x, y, x^{\prime}$ and $y^{\prime}$. By applying Fourier transformation and Parseval's theorem along the $x$-direction to the equation, then analytically integrating it along the $y$-direction, one finally yields the governing equation in the spectral domain:

$$
\int_{-\infty}^{\infty} \tilde{\mathbf{w}}\left(k_{x}\right)\left[\tilde{\mathbf{G}}\left(k_{x}, k_{z}\right)-\tilde{\mathbf{P}}\right] \tilde{\mathbf{J}}\left(k_{x}\right) d k_{x}=0
$$

Note that only single integration with respect to $k_{x}$ is involved in (2), thus it can be treated as the conventional spectral domain approach to find the phase and attenuation constants. In this study, the Legendre polynomials and piecewise linear functions are chosen as the $x$ - and $y$-dependent bases, respectively, for the unknown current $\mathbf{J}$.

To derive the matrix equation for the propagation constant, the Galerkin's method is used, in which the bases of $\mathbf{w}(x, y)$ are the same as those of $\mathbf{J}(x, y)$. Then the propagation constant $k_{z}=\beta-j \alpha$ can be found by solving the matrix equation.

\section{Numerical results}

To discuss the accuracy of the new approach's results, the frequency-dependent effective dielectric constants $\epsilon_{\text {eff }}=\left(\beta / k_{0}\right)^{2}$ are shown in Fig. 2 with those of [4] also included for comparison. Agreement in both results is observed with pointwise difference of at most 1 percent.

Fig. 3 presents a comparison of our results with those of [2] which is based on the modematching technique. There are some disagreement (about 2 percent) in effective dielectric constants, however the attenuation constants $\alpha$ agree well in each other. The disagreement may be caused by the fact that the structure for modematching analysis is closed while ours is open. In general, the phase constants for closed structures are less than those for open ones.

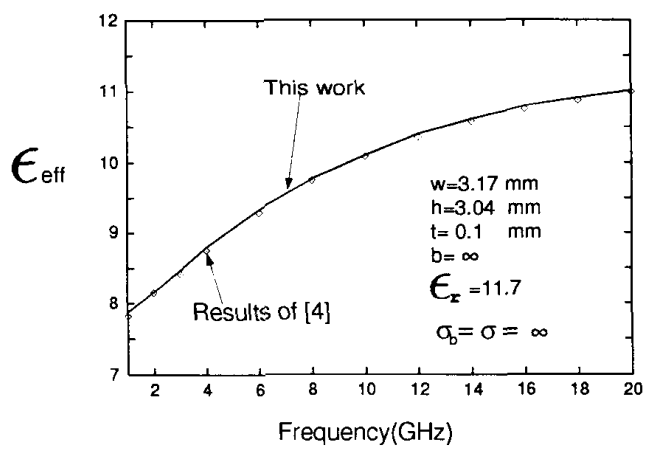

Figure 2: Effective dielectric constant $\epsilon_{\text {eff }}$ versus frequency .

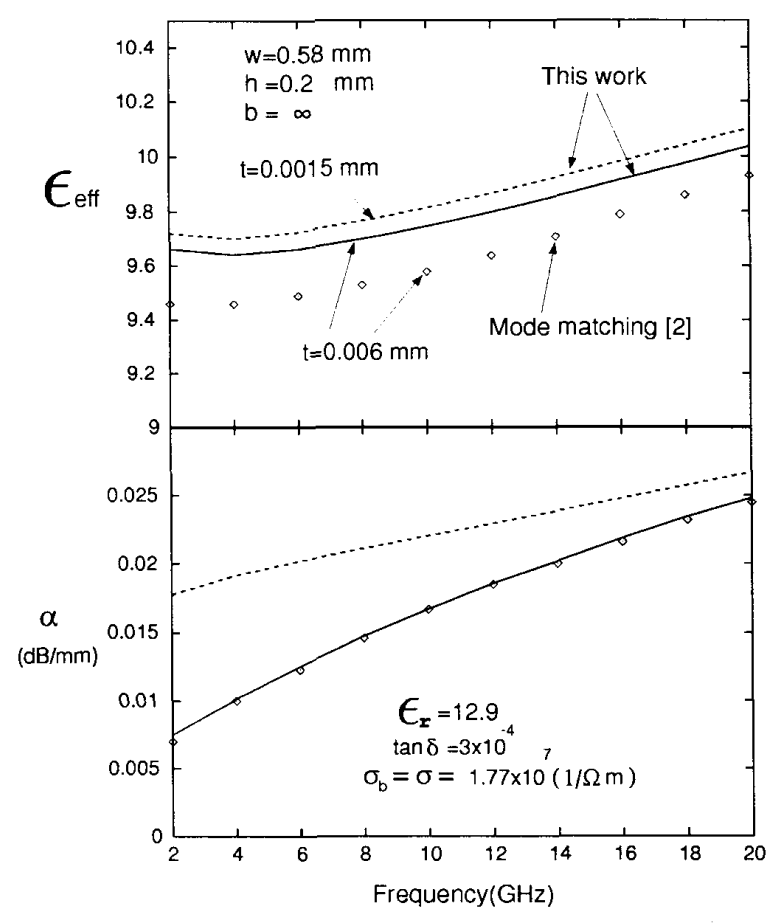

Figure 3: Effective dielectric constant $\epsilon_{\text {eff }}$ and attenuation constant $\alpha$ versus frequency. 
Shown in Fig. 4 is a comparison of the attenuation constants with the measured results of [5]. Good agreement between two results is also observed. It is interesting to note that the phase constant presents a minimum in the neighborhood of $17 \mathrm{GHz}$. Another thing should be emphasized is the negative slope in low frequency. When the conductivity $\sigma$ approaches infinity, the dispersive curve grows as the frequency increases( like Fig. 2 ), however when the conductivity $\sigma$ is finite, the skin depth in low frequency should not be treated as an infinitesimal value. The effect of finite conductivity in quasi-static range is important and should be carefully examined.

Shown in Fig. 5 is the distribution of axial current on the strip with $w=20 \mu \mathrm{m}$ and frequen$c y=7 \mathrm{GHz}$. As expected, the edge enhancement behavior is found for the axial current along $x$ and $y$-directions, but the edge current is finite instead of infinity. To discuss the skin effect phenomenon due to the finite conductivity, let us watch the curve $x=0$ in Fig. 5a. From $y=0$ to $y=0.625 t$, the magnitude is changed from 0.7 to 0.3 . This behavior is agreed well with the skin depth $\delta$ which is about $1 \mu \mathrm{m}$. On the other hand, the effective skin depth $\delta_{\text {eff }}$ which reads from Fig. $5 \mathrm{~b}$ along the curve $y=t / 2$ is about $2 \delta$. It is due to the fact that the thickness $t$ is much less than the width $w$ thus $\delta_{\text {eff }}$ would be great than $\delta$. This phenomenon was also observed by [6].

\section{Conclusions}

In this study, we propose a new method to deal with the microstrip line with layer structure in which the thickness and conductivity of signal strip are finite. The phase constant $\beta$ and attenuation constant $\alpha$ of the microstrip line with finite strip thickness and finite conductivity have been discussed, together with the current distributions along the signal strip. The advantage in the CPU time is reflected by the fact that the calculation of phase and attenuation constants per frequency point by [2] required 130 seconds using the IBM $3090-200 \mathrm{E}$, while our approach requires $30 \mathrm{sec}-$ onds using the SUN SPARCSTATION 2.

The proposed method can be applied to the structures with superconductor signal strip and/or semiconductor substrates. Besides, ex-

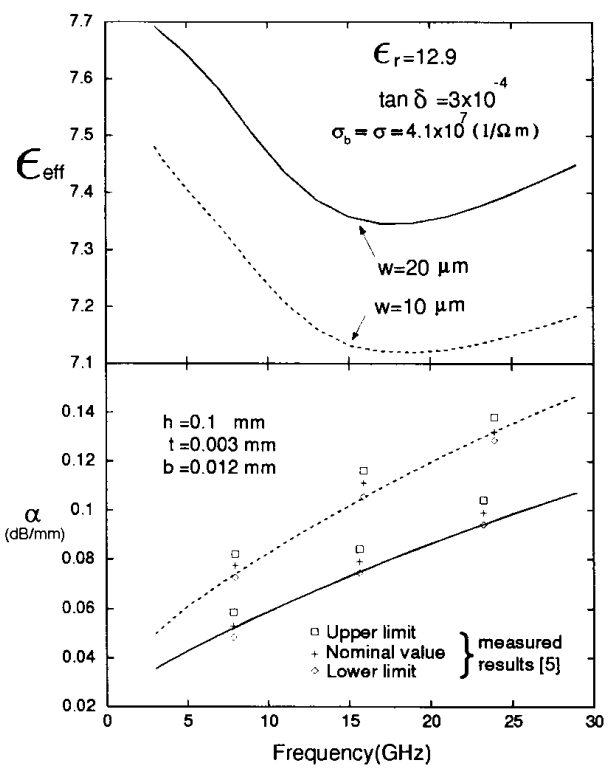

Figure 4: Effective dielectric constant $\epsilon_{\text {eff }}$ and attenuation constant $\alpha$ versus frequency.
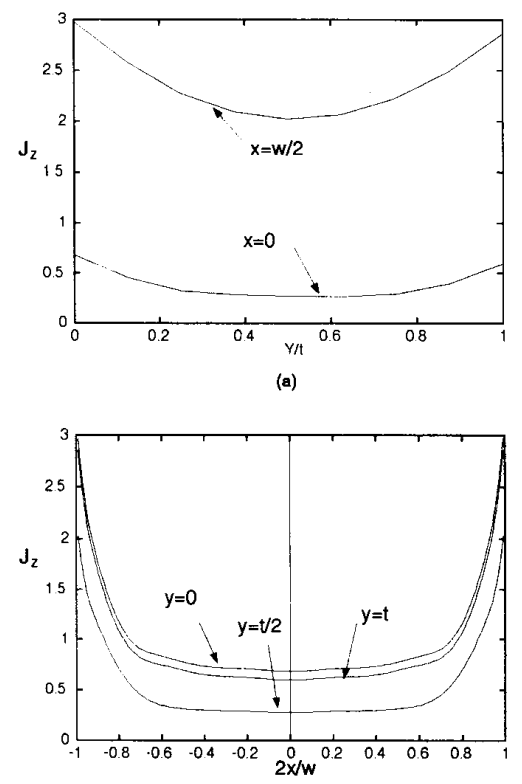

(b)

Figure 5: Current distributions along

(a) $y$-direction and

(b) $x$-direction. 
tension to the coplanar strips and coplanar waveguides is straightforward.

The disadvantage of the method is that the number of bases along $y$-direction is proportional to the thickness- skin depth ratio $t / \delta$. To avoid the increased CPU time as $t / \delta$ is increased, the nonuniform division along $y$-direction should be chosen.

\section{References}

[1] R. A. Pucel, D. J. Massé, and C. P. Hartwig, "Losses in Microstrip," IEEE Trans. Microwave Theory Tech., vol. 16, pp. 342-350, June 1968.

[2] W. Heinrich, "Full-wave analysis of conductor losses on MMIC transmission lines," IEEE Trans. Microwave Theory Tech., vol. 38, pp. 1468-1472, Oct. 1990.

[3] F. J. Schmückle and R. Pregla, "The method of lines for the analysis of lossy planar waveguides," IEEE Trans. Microwave Theory Tech., vol. 38, pp. 1473-1479, Oct. 1990.

[4] C. Shih, R. B. Wu, S. K. Jeng, and C. H. Chen, "Frequency-dependent characteristics of open microstrip lines with finite strip thickness," IEEE Trans. Microwave Theory Tech., vol. 37, pp. 793-795, April 1989.

[5] M. Goldfarb and A. Platzker, "Losses in GaAs microstrip," IEEE Trans. Microwave Theory Tech., vol. 38, pp. 1957-1963, Dec. 1990.

[6] W. Heinrich, "Conductor loss on transmission lines in monolithic microwave and millimeter-wave integrated circuits," International Journal of Microwave and Millimeter-Wave Computer-Aided Engineering, vol. 2, no. 3, pp. 155-167, 1992. 